\title{
Study of Freeze-dried Chitosan Beads Encapsulating Live Lactic Acid Bacteria for Removal of Reactive Dyes *
}

\author{
Chi Him Jim Luk ${ }^{a}$, Joanne Yip ${ }^{\text {a,*, }}$ Chun Wah Marcus Yuen ${ }^{\mathrm{a}}$ \\ Kim Hung Lam ${ }^{b}$, Chi Wai Kan ${ }^{a}$ \\ ${ }^{a}$ Institute of Textiles and Clothing, The Hong Kong Polytechnic University, Hung Hom, Hong Kong \\ ${ }^{\mathrm{b}}$ Department of Applied Biology and Chemical Technology, The Hong Kong Polytechnic University \\ Hung Hom, Hong Kong
}

\begin{abstract}
Water pollution by the discharge of dye residue has been polluting the planet for decades. Chitosan is one of the natural abundant biomasses that show excellent adsorption toward colorants. Nevertheless, sophisticated chemical modifications of chitosan are inevitable to enhance physical and chemical stabilities in practical applications. In the present study, simple freeze-drying has been adopted to chitosan (CHI) beads showing good stability at low levels of $\mathrm{pH}$. The results show complete decolourisation of Reactive Blue 19 (RB19) within 2 hours at pH 3 and temperature of $37^{\circ} \mathrm{C}$. The encapsulation of live bacterium Lactobacillus casei (L. casei) into chitosan beads for dyestuff removal is also evaluated. Kinetics studies show a faster initial rate of adsorption by $L$. casei-chitosan beads at $\mathrm{pH} 3$ and temperature of $37^{\circ} \mathrm{C}$. The adsorptions partially follow the intraparticle diffusion mechanism.
\end{abstract}

Keywords: Chitosan Beads; Freeze-drying, Lactobacillus Casei; Reactive Blue 19; Removal

\section{Introduction}

Water pollution is one of the key issues in the world. Besides organic pollutants and heavy metal ions, textile dyes are one of the pollutants that are causing serious environmental problems. A few ppm scale concentration of dyes already expresses high colour intensity which alters the light penetration to the aqua ecosystem [1]. The pollution spreads to agriculture and humans in developing countries without proper water treatment facilities. Since decades ago, research has been focusing on the use of environmentally friendly and cost effective biomasses to remove polluting colourants in water [2]. Among numerous biomasses (plant origins, industrial wastes, fruits peels and pits, etc.), chitosan has shown an excellent adsorption capacity to synthetic dyes, giving $10^{2}$ to even $10^{3} \mathrm{mg} \mathrm{g}^{-1}$ of removal [3]. However, since chitosan dissolves in acidic medium,

\footnotetext{
${ }^{\star}$ The work was supported by a Hong Kong Polytechnic University research grant [A/C U893] and RGC General Research Fund [A/C B-Q26S].

${ }^{*}$ Corresponding author.

Email address: tcjyip@polyu.edu.hk (Joanne Yip).
} 
chemical modifications are usually required to maintain its chemical and physical stabilities during the adsorption process. The modifying partners are usually bi-functional and reactive toward the - $\mathrm{NH}_{2}$ group in chitosan, such as glutaraldehyde, epichlorohydrin, etc. The modification processes are sometimes complicated and might involve toxic chemicals.

Another popular type of adsorbent is microbial biomass [4]. The application of bacteria and fungi in the removal of water pollutants has been intensively studied for years. The microbial cells adsorb synthetic dyes by the functional groups on the cell wall and chemical modifications can even enhance the adsorption capacity. Although excellent biosorption capacity can be achieved, the separation of microorganisms after water treatment in industrial plants remains problematic as clogging of parts might occur [5]. Moreover, the harsh bulk environments are threatening if it is necessary for the microorganisms to be alive during the water treatment process.

In the present study, an environmentally safe ionotropic crosslinking agent, sodium triphosphate penta-basic (NaTPP), has been used to form chitosan beads followed by a simple freeze-drying process. Freeze-drying the chitosan beads stabilizes the viability of the encapsulated bacterium (Lactobacillus casei) during storage or before any adsorption process. Immobilization of the microorganisms by polymeric supports is one of the most effective methods to solve the problems in the industry while maintaining satisfactory adsorption capacity toward pollutants.

\section{Experimental}

\subsection{Chemicals}

Chitosan (practical grade, from shrimp shells, deacetylation $\geq 75 \%$ ), sodium triphosphate pentabasic ( $\geq 98 \%$ ), potassium bromide (KBr, FTIR grade), and Remazol Brilliant Blue R (Reactive Blue 19, dye content 50\%) were obtained from Sigma-Aldrich LLC. Glacial acetic acid was obtained from VWR International LLC. Fig. 1 shows the molecular structure of Reactive Blue 19.

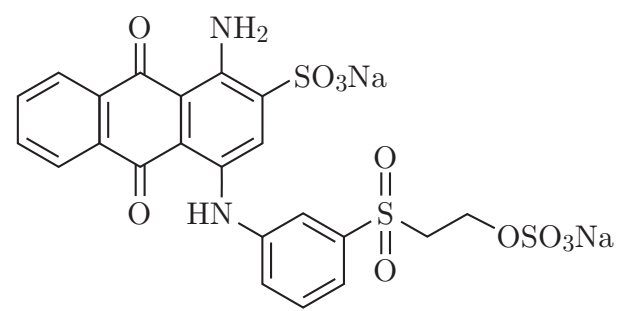

Fig. 1: Molecular structure of Reactive Blue 19 (RB19)

\subsection{Microorganism and Culturing}

Lactobacillus casei (L. casei, ATCC 393) was obtained from ATCC Co. The culture medium (de Man, Rogosa and Sharpe (MRS)) broth and agar were obtained from BD Co. The bacterium was rehydrated and inoculated in accordance with standard procedures. Cultured L. casei was harvested after inoculation of 5 days at a temperature of $37^{\circ} \mathrm{C}$ followed by 10 minutes of centrifugation at $6000 \mathrm{rpm}$ under a temperature of $4{ }^{\circ} \mathrm{C}$. The bacterium pellets were subjected 
to freeze-drying at $0.120 \mathrm{mBar}$ under a temperature of $-51{ }^{\circ} \mathrm{C}$ for 24 hours (Labconco, FreeZone $2.5 \mathrm{~L}$ freeze dryer). The freeze-dried bacterium was then stored at $4{ }^{\circ} \mathrm{C}$ for experimental use.

\subsection{Preparation of Chitosan Beads}

Chitosan (CHI, 1.5\% (w/v)) was prepared with $1.5 \%(\mathrm{v} / \mathrm{v})$ glacial acetic acid in deionized water until complete dissolution. The solution was then centrifuged at $4000 \mathrm{rpm}$ for 10 minutes and the supernatant was gathered for use. A $2 \%(\mathrm{w} / \mathrm{v})$ sodium tripolyphosphate (NaTPP) solution was prepared with deionized water followed by $\mathrm{pH}$ adjustment by $\mathrm{HNO}_{3}$ to a $\mathrm{pH}$ of 6-7. The CHI solution was transferred into a syringe equipped with a $25 \mathrm{G}$ needle tip. The CHI solution was added drop wise at $10 \mathrm{ml}$ each time into $50 \mathrm{ml}$ of the NaTPP solution with a tip-solution surface distance of approximately $4 \mathrm{~cm}$ (Fig. 2). CHI beads immediately formed after contact with the NaTPP bath. The beads were post-hardened for 60 minutes.

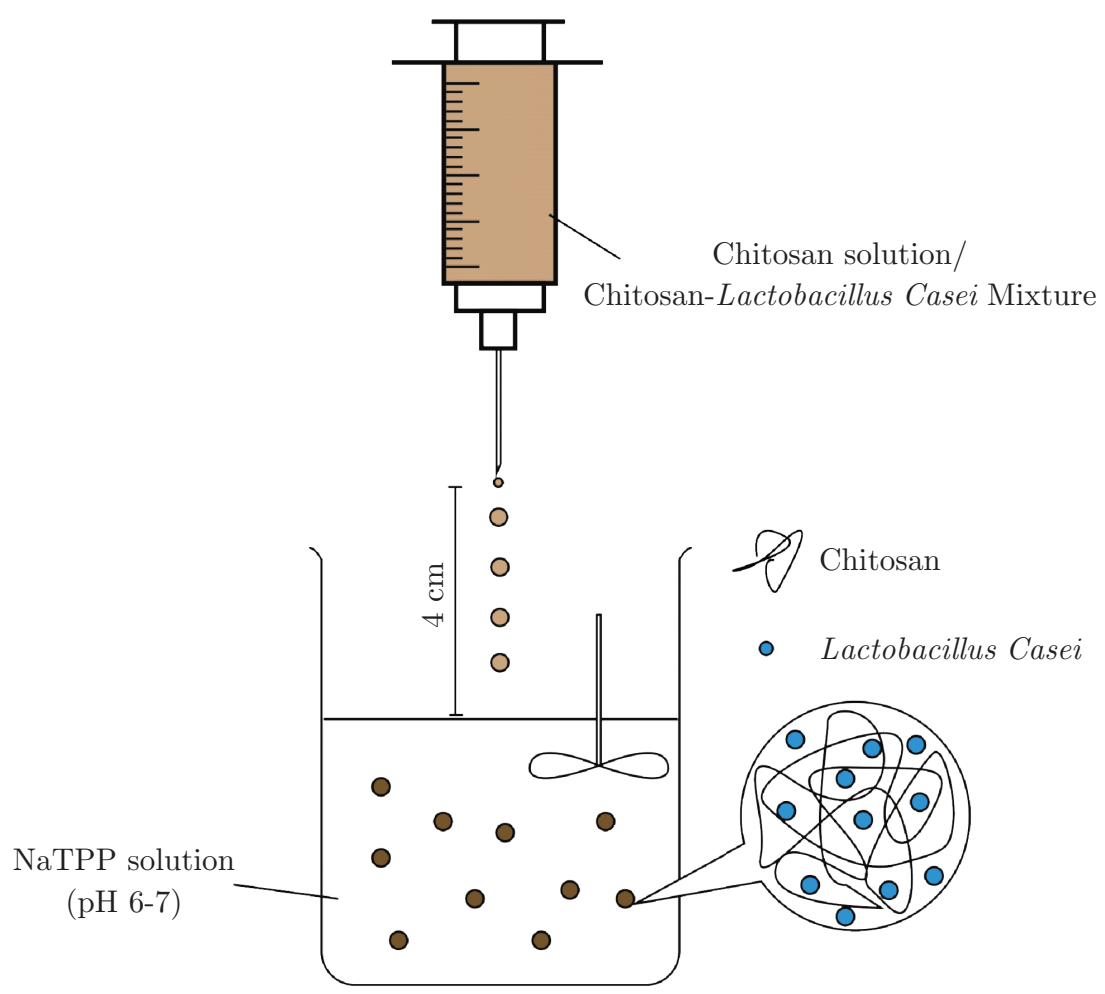

Fig. 2: Illustration of preparation of TPPCB (adding of CHI solution) and MTB (adding of CHILactobacillus casei mixture)

For the preparation of the L. casei-encapsulated CHI beads (MTB), freeze-dried L. casei was first rehydrated in the MRS broth and then mixed with the CHI solution at a volume ratio of 1:9. The final concentration was maintained in a $1.5 \%(\mathrm{w} / \mathrm{v})$ CHI solution in $1.5 \%(\mathrm{v} / \mathrm{v})$ glacial acetic acid with $0.2 \%(\mathrm{w} / \mathrm{v})$ L. casei. The mixture was then dipped into the NaTPP solution as mentioned above.

Both the blank and L. casei-encapsulated CHI beads were brought to post-inoculation in the MRS broth for 24 hours. The beads were then filtered and rinsed with deionized water. The harvested beads were then subjected to freeze-drying at 0.120 mBar under a temperature of -51 
${ }^{\circ} \mathrm{C}$ for a further 24 hours (Labconco, FreeZone 2.5 L freeze dryer). The freeze-dried blank CHI (TPPCB) and L. casei-encapsulated CHI (MTB) beads were stored in a desiccator before use. Fig. 3 shows the appearance of the MTB and TPPCB. The MTB has a deeper yellow colour in comparison to the TPPCB which is caused by the encapsulation of $L$. casei.

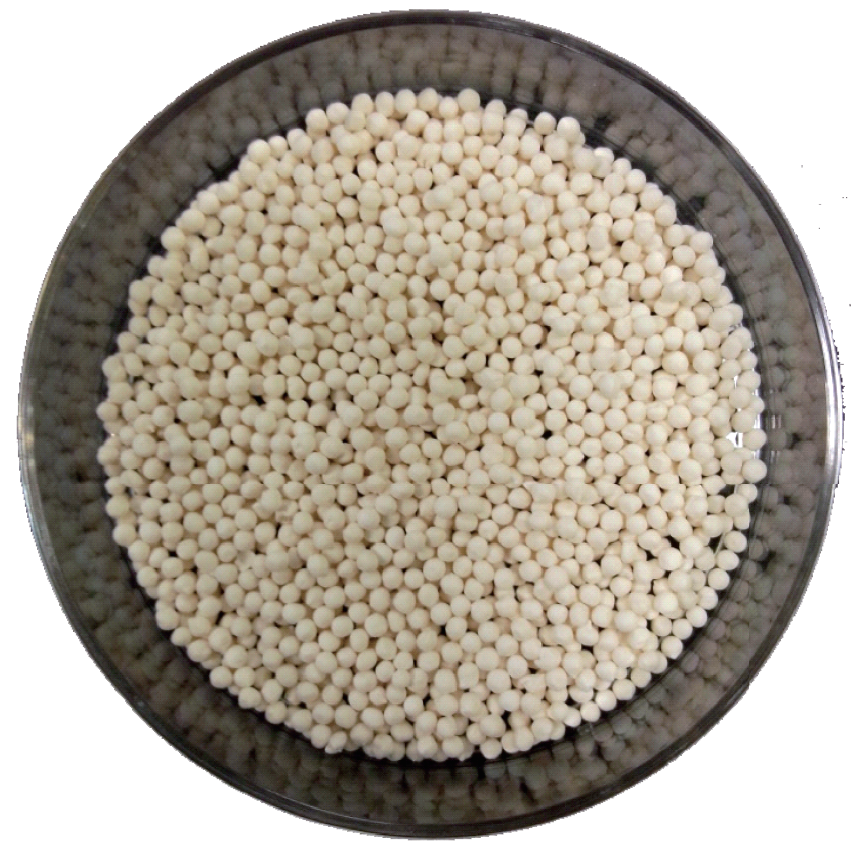

(a)

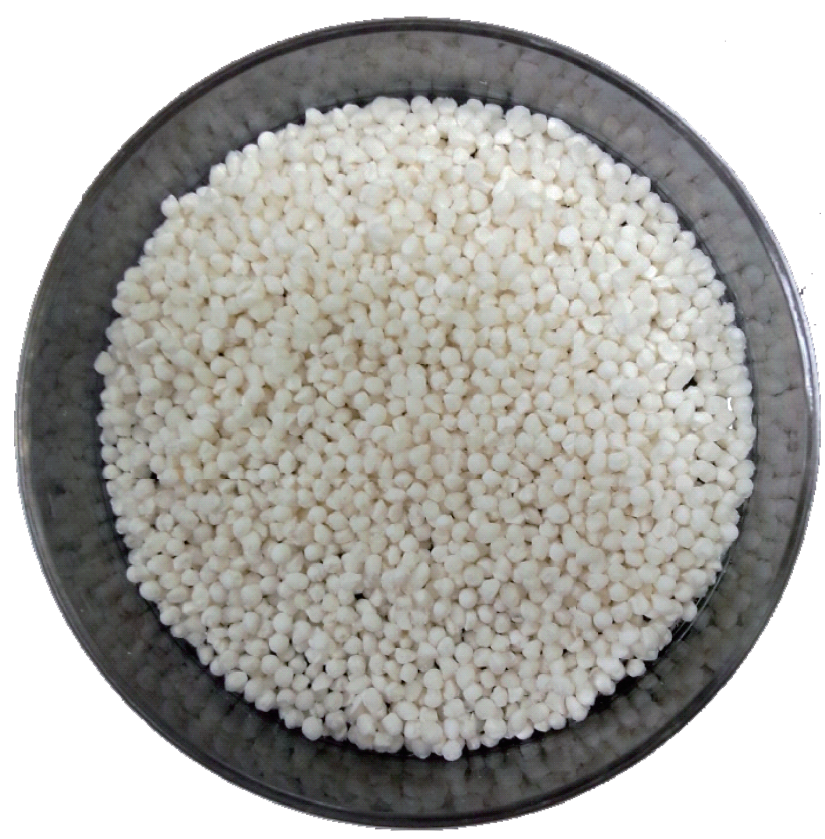

(b)

Fig. 3: MTB (a) and TPPCB (b). MTB shows a deeper yellow colour than TPPCB which was caused by the encapsulation of $L$. casei

\subsection{Estimation of Viability of Encapsulated L. casei}

After the preparation of the MTB and TPPCB, a $20 \mathrm{mg}$ sample of each item was weighed, grinded and rehydrated in the MRS broth solution. A serial dilution of each mixture was conducted and then transferred to an MRS agar plate for 5 days of inoculation under a temperature of $37^{\circ} \mathrm{C}$. The viability of the encapsulated $L$. case $i$ was expressed as the $\mathrm{CFU} / \mathrm{g}$ of the MTB and the encapsulated amount was expressed as the mg L. casei/g MTB by the mass balance.

\subsection{Zeta Potential Measurement at Different pH}

Surface charge distributions of adsorbents at different $\mathrm{pH}$ contribute much to the adsorption of synthetic dyes. In this study, zeta potential is used for quantification of the charge magnitude. TPPCB, MTB and L. case $i$ were grounded and mixed with $100 \mathrm{ml}$ of deionized water respectively with a concentration of $0.01 \mathrm{~g} \mathrm{ml}^{-1}$. The mixtures were stirred overnight at $1000 \mathrm{rpm}$ and then subjected to zeta potential measurement with a ZetaPlus zeta potential analyser (BrookHaven Instrument Co.). Before each measurement, the suspensions were adjusted to pre-assigned $\mathrm{pH}$ values by various concentrations of nitric acid $\left(\mathrm{HNO}_{3}\right)$ and sodium hydroxide $(\mathrm{NaOH})$ solutions respectively without the addition of background electrolytes. 


\subsection{Fourier Transform Infrared (FTIR) Spectroscopy}

The Fourier transform infrared (FTIR) spectra of the TBBCB, MTB and L. casei were obtained by using a Perkin-Elmer Spectrum 100 FTIR spectrometer. Dried-pressed pellets were obtained by grinding different samples with FTIR grade $\mathrm{KBr}$ with an agate mortar.

\subsection{Batch Adsorption Experiment}

The effect of $\mathrm{pH}$ on the adsorption of Reactive Blue (RB19) by TPPCB was examined. A RB19 solution $(0.15 \mathrm{mM}, 50 \mathrm{ml})$ was prepared by diluting a $2.5 \mathrm{mM}$ stock solution and the desired $\mathrm{pH}$ values were adjusted by using $1 \mathrm{M} \mathrm{HNO}_{3}$ and $\mathrm{NaOH}$. Before the adsorption experiments, the dye solutions were first warmed in a $37^{\circ} \mathrm{C}$ water bath. The adsorbent $(0.1 \mathrm{~g})$ was then weighed and transferred to the pre-warmed dye solution, and the shaking bath was maintained at 140 rpm (linear shaking). The $\mathrm{pH}$ of the dye solutions were adjusted by using $1 \mathrm{M} \mathrm{HNO}_{3}$ and $\mathrm{NaOH}$ throughout the adsorption. Aliquot $(100 \mu \mathrm{l})$ was withdrawn at pre-determined time intervals and accordingly diluted. The dye concentration was determined by using a Perkin Elmer Lambda 18 UV-VIS spectrophotometer at $592 \mathrm{~nm}$. The decolourisation \% was determined by Eq. (1):

$$
\text { decolourisation } \%=\frac{C_{0}-C}{C_{0}} \times 100 \%
$$

where $C_{0}$ is the initial concentration of the dye solution in $\mathrm{mM}$ and $C$ is the concentration of the dye at a pre-determined time interval.

Kinetics experiments were performed by transferring $0.1 \mathrm{~g}$ of adsorbent (MTB/TPPCB) into $50 \mathrm{ml}$ of $1.5 \mathrm{mM} \mathrm{RB} 19$ dye solution at $37^{\circ} \mathrm{C}$ under a $\mathrm{pH}$ of 3 . The adsorbed amount of RB19 at time $t, q_{t}$, was determined by Eq. (2):

$$
q_{t}=\frac{C_{0}-C_{t}}{M} \times V
$$

where $C_{0}$ is the initial concentration of the dye solution in $\mathrm{mM}$ and $C_{t}$ is the concentration of the dye at a pre-determined time interval, $M$ is the mass of the adsorbent and $V$ is the volume of the dye solution.

\section{Results and Discussion}

\subsection{Encapsulation of $L$. casei}

After the encapsulation of $L$. casei into the MTB, the amount of $L$. casei was increased by further inoculation in the MRS broth. It was found that the amount of $L$. casei was approximately 200 $\mathrm{mg}$ L. casei/g MTB while the viability is as high as $2.13 \times 10^{9} \mathrm{CFU} / \mathrm{g} \mathrm{MTB}$, which was comparable to similar work by Vodnar et al. where the CHI beads were not freeze-dried [6]. This shows that after the encapsulation process, post-inoculation and freeze-drying, the viability of $L$. casei can be maintained at a high value. The preparation process variables, such as the $\mathrm{pH}$ and chemicals, did not induce a significant amount of harmful effects toward the bacteria. Therefore, it is possible for further biosorption based on live biomass. 


\subsection{Zeta Potential Measurement}

Fig. 4 shows the zeta potential measurement of TPPCB, MTB and L. casei respectively at different $\mathrm{pH}$ levels, which correlates to the surface charge distributions. Three of them showed similar trend with more positive zeta potential at low $\mathrm{pH}$ and less positive or negative value at high $\mathrm{pH}$. The isoelectric point of TPPCB was around 7.5 while MTB has an isoeletric point of 6.8, and $L$. casei has an isoelectric point near $\mathrm{pH}$ 3.8. Chitosan is composed of $N$-glucosamine and $N$-acetylglucosamine. The polysaccharide possesses numerous of hydroxyl group, amino group and acetyl group. These functional groups easily interact with protons and hydroxides when subjected to different $\mathrm{pH}$ environments. Therefore TPPCB and MTB has a high positive surface charge when subjected to low $\mathrm{pH}$ environment and vice versa, resulting in positive and negative zeta potentials respectively. On the other hand, it was interesting to observe the low isoelectric point near pH 3.8 when measuring the zeta potential of $L$. casei. It implies that the microbial cells possesses numerous negative-charged functional groups on the cell wall, hence the net surface charge was neutral around $\mathrm{pH} 3.8$ and became net negative when the $\mathrm{pH}$ became higher. Moreover, the zeta potential of MTB was overall less positive than TPPCB. It might be due to the combination with $L$. case $i$ which suppresses the overall surface charge.

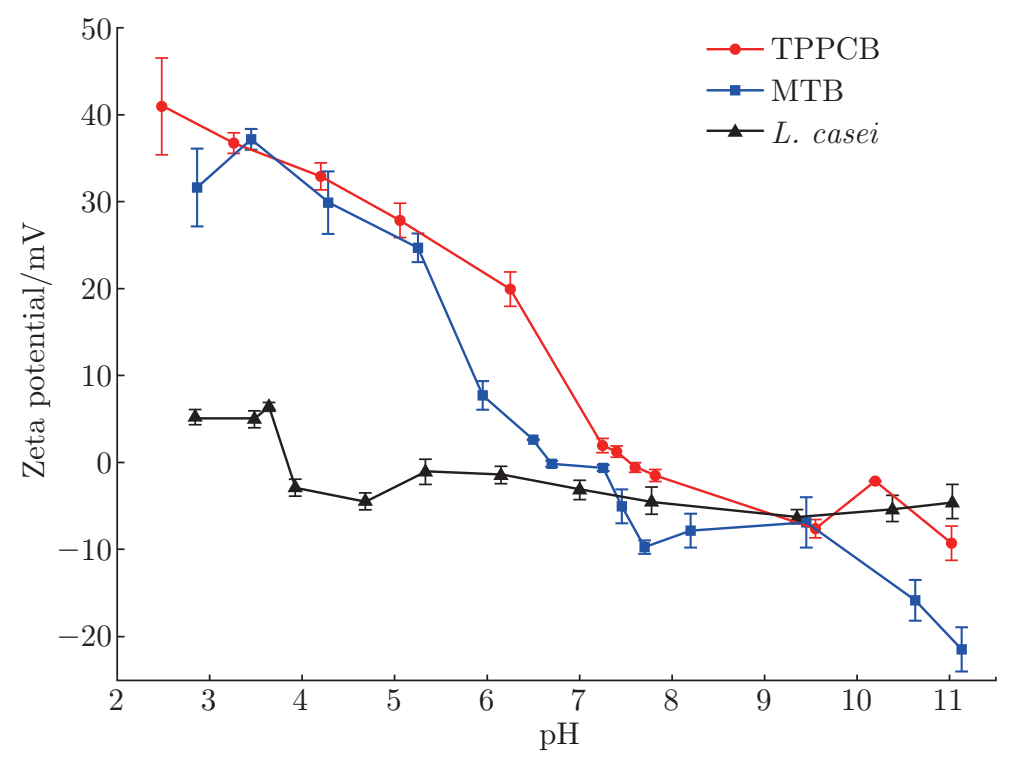

Fig. 4: Zeta Potential measurement of (a) TPPCB, (b) MTB and (c) L. casei

\subsection{FTIR Spectroscopy}

Fig. 5 shows the FTIR spectra of (a) MTB, (b) TPPCB and (c) L. casei. Table 1 shows the absorption bands with regards to the corresponding functional groups. L. case $i$ is a gram-positive bacteria with a cell wall that has a high proportion of peptidoglycan in the outer layer [7]. The peptidolycan layer contains a polymeric network of $N$-acetylglucosamine and $N$-acetylmuramic acids. On the other hand, CHI is composed of glucosamine and $N$-acetylglucosamine and the proportion depends on the degree of deacetylation [8]. Therefore, the three spectra share common absorption bands in terms of the presence of glucosamine and $N$-acetylglucosamine units (amido, amino, ether and alcoholic absorption bands). Both the TPPCB and MTB show the presence 


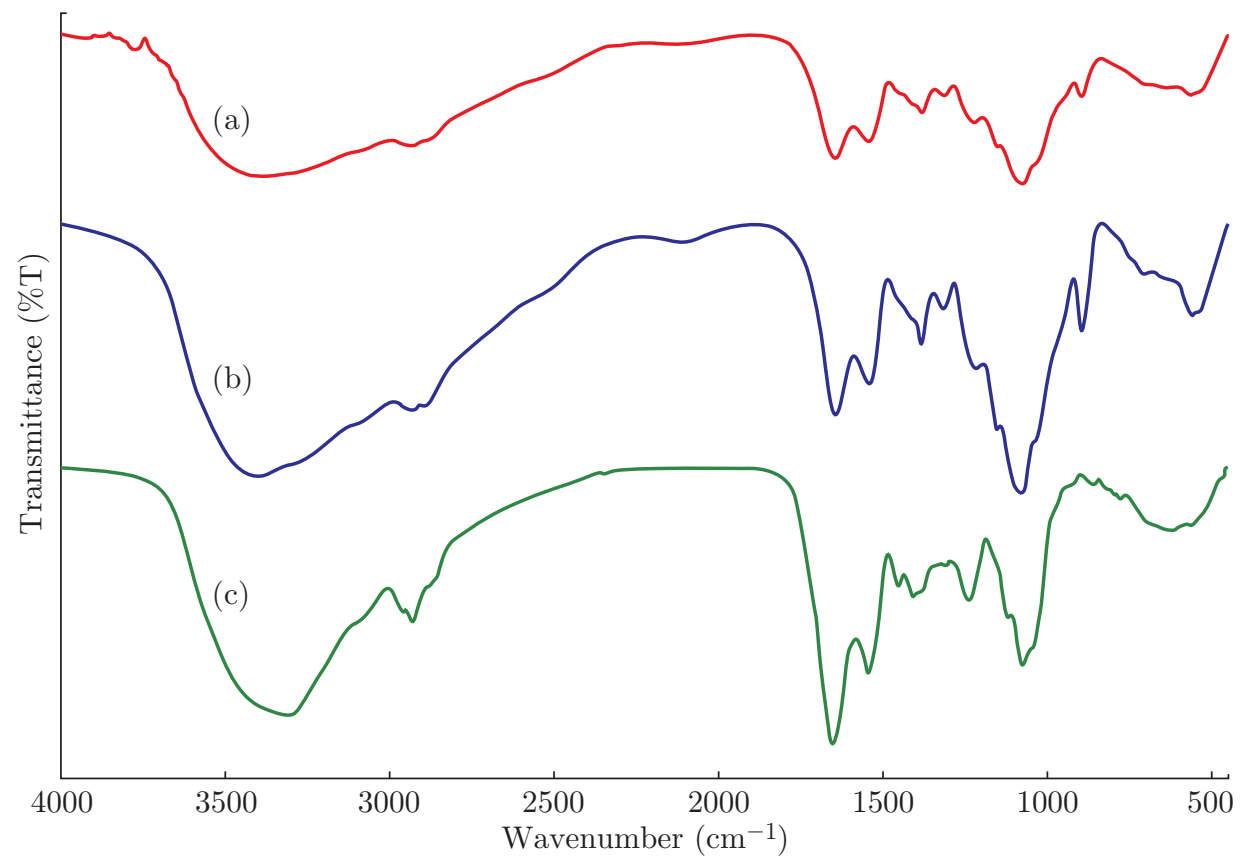

Fig. 5: FTIR spectra: (a) MTB, (b) TPPCB and (c) L. casei

Table 1: Designation of functional groups to FTIR absorption bands of $L$. casei, TPPCB and MTB

\begin{tabular}{|c|c|c|c|c|}
\hline Absorption band $\left(\mathrm{cm}^{-1}\right)$ & Designated functional groups & L. casei & ТРРСВ & MTB \\
\hline 895 & $\mathrm{~N}-\mathrm{H}$ bending (amines) & - & + & + \\
\hline 1076 & $\begin{array}{c}\mathrm{C}-\mathrm{O}-\mathrm{C} \text { stretching } \\
\text { (dialkyl ethers) }\end{array}$ & + & + & + \\
\hline $1119-1222$ & $\begin{array}{c}\mathrm{P}=\mathrm{O} \\
\text { (triphosphate) }\end{array}$ & $+(1$ band $)$ & $+(2$ bands $)$ & $+(2$ bands $)$ \\
\hline 1238 & $\begin{array}{l}\mathrm{C}-\mathrm{O} \text { stretching } \\
\text { (carboxylic acid) }\end{array}$ & + & - & - \\
\hline $1315-1381$ & $-\mathrm{CH}_{3}$ bending (amide) & + & + & + \\
\hline $1400-1460$ & $\begin{array}{c}\mathrm{O}-\mathrm{H} \text { bending } \\
(\text { carboxylic acid })\end{array}$ & + & - & - \\
\hline 1545 & $\mathrm{~N}-\mathrm{H}$ bending (amine) & + & + & + \\
\hline 1645 & $\mathrm{C}=\mathrm{O}$ stretching (amide) & + & + & + \\
\hline 2930 & $\mathrm{C}-\mathrm{H}$ stretching (alkane) & + & + & + \\
\hline $3300-3400$ & $\mathrm{O}-\mathrm{H}$ stretching (alcohol) & + & + & + \\
\hline
\end{tabular}

of $\mathrm{N}-\mathrm{H}$ bending at $895 \mathrm{~cm}^{-1}$ while $L$. casei did not. This is due to the presence of primary amino groups on CHI. Another common absorption band is related to the presence of $\mathrm{P}=\mathrm{O}$, which is responsible for the presence of phosphodiester bonds on L. casei [7] and NaTPP in TPPCB and MTB after ionotropic crosslinking. However, it can be observed that there are two $\mathrm{P}=\mathrm{O}$ absorption bands in the FTIR spectra of TPPCB and MTB (overlapped with the $\mathrm{C}-\mathrm{O}-\mathrm{C}$ absorption band), but one in that of $L$. casei. A possible reason is due to the presence of different $\mathrm{P}=\mathrm{O}$ planes on the NaTPP ion after the crosslinking reaction (different planes of 
$\mathrm{P}=\mathrm{O})$ while the phosphodiester bond only has one plane of $\mathrm{P}=\mathrm{O}$ bond. Due to the presence of $N$-acetylmuramic acid, the spectrum of $L$. casei showed the presence of carboxylic $\mathrm{O}-\mathrm{H}$ bending while this was not found in the spectrum of TBBCB and MTB.

\section{4 pH Effect on the Removal of RB19 by TPPCB}

Fig. 6 shows the adsorption of RB19 by TPPCB at $\mathrm{pH}$ of 3,5.5 and 9 . The initial rate of adsorption is not affected much by the changes in the $\mathrm{pH}$ while this is considerably different for the adsorption equilibriums. Under both $\mathrm{pH} 3$ and 9, a higher adsorption equilibrium is evident as opposed to $\mathrm{pH}$. The adsorption equilibrium of TPPCB at $\mathrm{pH} 3$ is the highest $(\geq 99 \%)$.

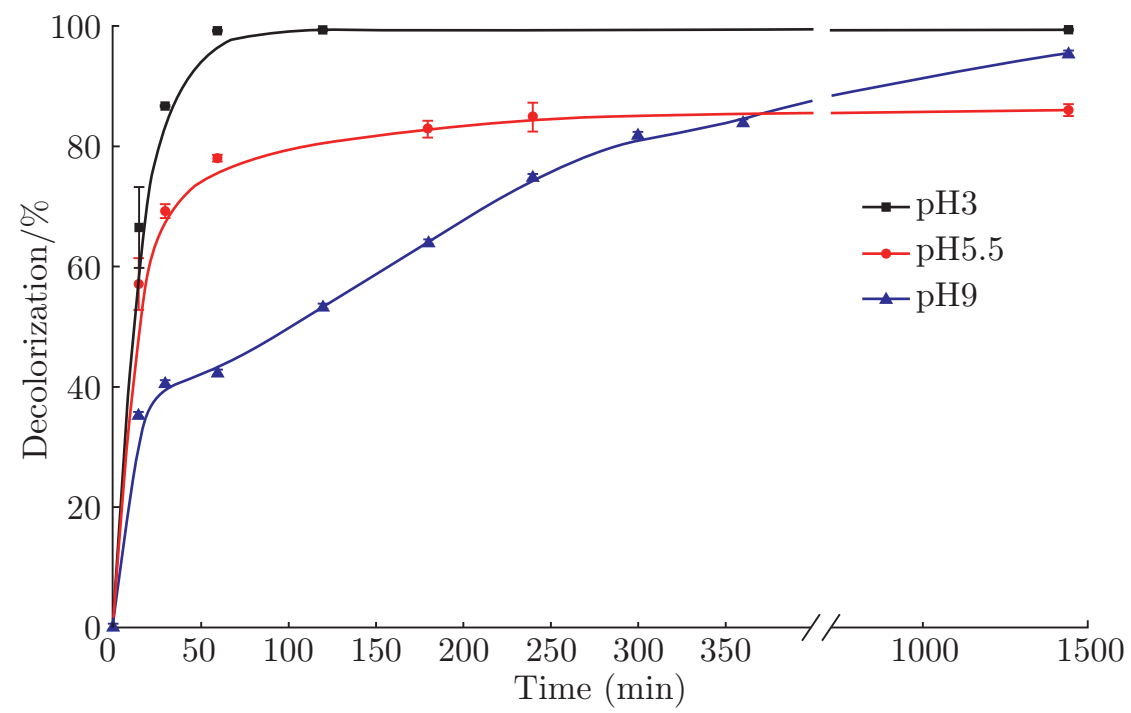

Fig. 6: $\mathrm{pH}$ effect of removal of RB19 by TPPCB. Adsorption conditions: temperature of $37{ }^{\circ} \mathrm{C}, 50 \mathrm{ml}$ of $0.15 \mathrm{mM}$ adsorbate, $0.1 \mathrm{~g}$ adsorbent, shaking speed of $140 \mathrm{rpm}$

The adsorption performance of particular synthetic dyes is closely related to the adsorbateadsorbent interactions under different $\mathrm{pH}$ environments. The adsorption capacity of TPPCB follows the trend of corresponding zeta potential except that at $\mathrm{pH}$ 9. At low $\mathrm{pH}$ levels, TPPCB interacts with bulk protons, hence there is a net positive surface charge. On the other hand, the RB19 dye molecules exist as their conjugate base. As a result, positively charged TPPCB would have a relatively strong interaction with anionic RB19 dye molecules. From this point of view, a higher bulk $\mathrm{pH}$ would give less positive surface charge to TPPCB, hence there is less effective adsorption of RB19 by TPPCB. Therefore, the adsorption equilibrium dropped at $\mathrm{pH}$ 5.5 to around $82 \%$ compared to $\mathrm{pH} 3$. Nevertheless, the adsorption equilibrium at $\mathrm{pH} 9$ was unexpectedly high which is comparable to $\mathrm{pH} 3$. It can be observed that there was a shoulder around $40 \%$ adsorption at a time of approximately $50 \mathrm{~min}$, which follows the trend of adsorption decrement upon increasing the bulk $\mathrm{pH}$. However, later increments of adsorption were observed until the equilibrium adsorption reached approximately 95\%. At a high $\mathrm{pH}$, the RB19 molecules were completely ionized and existed in its conjugate base form while TPPCB had a net negative surface charge surrounded by hydroxide ions. Normally, an opposite charge repulsion would mean suppressed adsorption. One possible reason is the presence of hydrogen bonding between TPPCB and RB19 dye molecules which have amino and anthraquinone groups (molecular structure of RB19 is shown in Fig. 1). Further confirmation of the adsorption mechanism would require the 
implementation of desorption experiments to understand the interaction between the dyes and TPPCB adsorbent.

\subsection{Adsorption Kinetics of MTP and TPPCB}

The application of different kinetics models provides more information on the adsorption mechanism, including the adsorption rate and the rate determining steps. After the study of $\mathrm{pH}$ effect on the removal of RB19 by TPPCB, the optimum $\mathrm{pH}$ was applied to both TPPCB and MTB and the pseudo first order [9], pseudo second order [10] and intraparticle diffusion [11] models were fitted to observe the removal mechanism. Table 2 shows the linear form of the three kinetic models.

Table 2: Linear forms of intraparticle diffusion, pseudo first and second order model. $k_{i}, k_{1}$, and $k_{2}$ are the rate constant of corresponding kinetic model; $q_{e}$ is the amount of adsorbed dye molecules at equilibrium and $q_{t}$ is the amount of dye adsorbed at time $t$

\begin{tabular}{cc}
\hline Kinetic models & Linear form \\
\hline Intraparticle diffusion model & $q_{t}=k_{i} t^{0.5}$ \\
Pseudo first order model & $\log \left(q_{e}-q_{t}\right)=\log q_{e}-\frac{k_{1}}{2.303} t$ \\
Pseudo second order model & $\frac{t}{q_{t}}=\frac{1}{k_{2} q_{e}^{2}}+\frac{1}{q_{e}} t$ \\
\hline
\end{tabular}

Fig. 7 shows the plots of (a) Removal of RB19 by MTB and TPPCB, kinetic models fitting of (b) Pseudo first order model, (c) Second order model and (d) Intraparticle diffusion model respectively and Table 3 summarizes the kinetics parameters of the modelling.

In the plot of RB19 removal versus time, the initial rate and overall $q_{t}$ of MTB were respectively faster and higher than those of TPPCB upon the removal of RB19. Nevertheless, there was not much difference in the $q_{e}$. When the adsorptions were fitted with the pseudo first order model, MTB and TPPCB showed an $\mathrm{R}^{2}$ of 0.9791 and 0.9794 respectively with similar rate constants $\left(0.0030 \mathrm{~min}^{-1}\right.$ for MTB and $0.0026 \mathrm{~min}^{-1}$ for TPPCB). On the other hand, when the two adsorptions were fitted with the pseudo second order model, the $\mathrm{R}^{2}$ of MTB was 0.9894 which showed a higher degree of agreement while TPPCB was less fitting to the model (0.9647). From the plot of $q_{t}$ against $t^{0.5}$, the intraparticle diffusion modelling showed multi-linearity on the adsorption by MTB, and TPPCB showed multi-step kinetics. The high values of $\mathrm{R}^{2}$ in the initial step $(>0.99)$ were attributed to the adsorbent surface adsorption while those in the equilibrium stage could be regarded as inner pore diffusion. In the mid-stage of adsorption, the correlation coefficients of the intraparticle diffusion fitting were 0.9549 for MTB and 0.9016 for TPPCB. Although the correlation coefficients were not satisfactory, the passing through of the origin of the two fittings indicated a possibility of an intraparticle diffusion mechanism in the mid-stage of absorption. On the other hand, when the two adsorptions were subjected to fitting with the pseudo first and second order models, high linearity was demonstrated in the mid-stage of absorption, starting from $t=90 \mathrm{~min}$. For both MTB and TPPCB, a further confirmation of the varying initial concentration of the kinetics will give a clearer indication whether the adsorption mechanism follows a chemisorption or physisorption mechanism. 


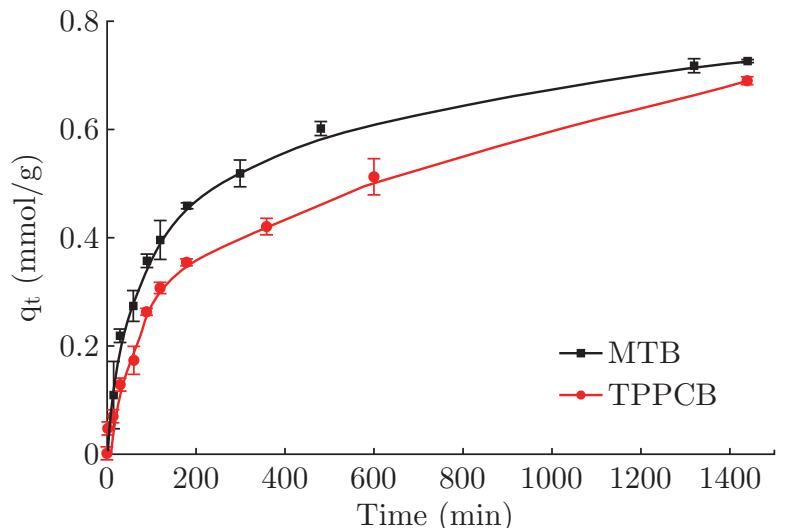

(a)

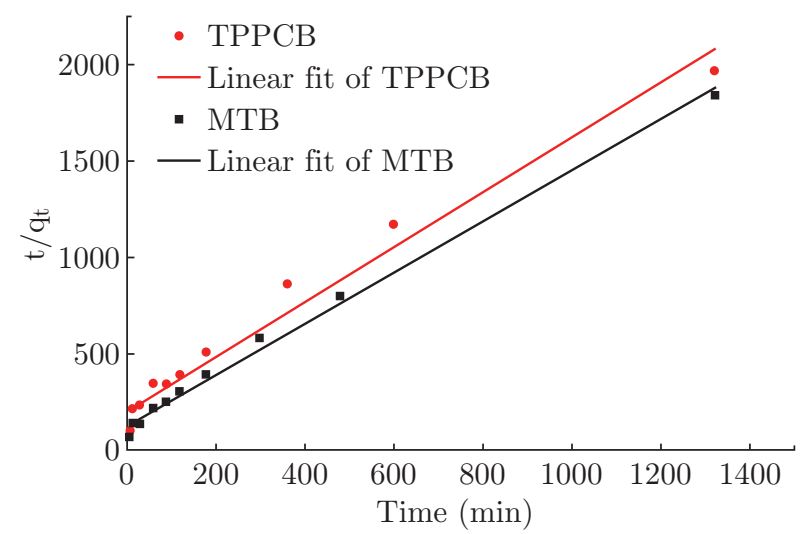

(c)

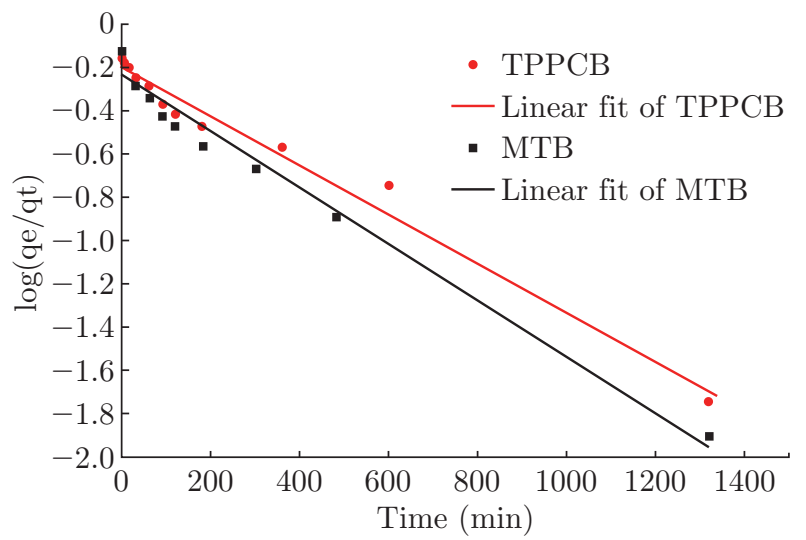

(b)

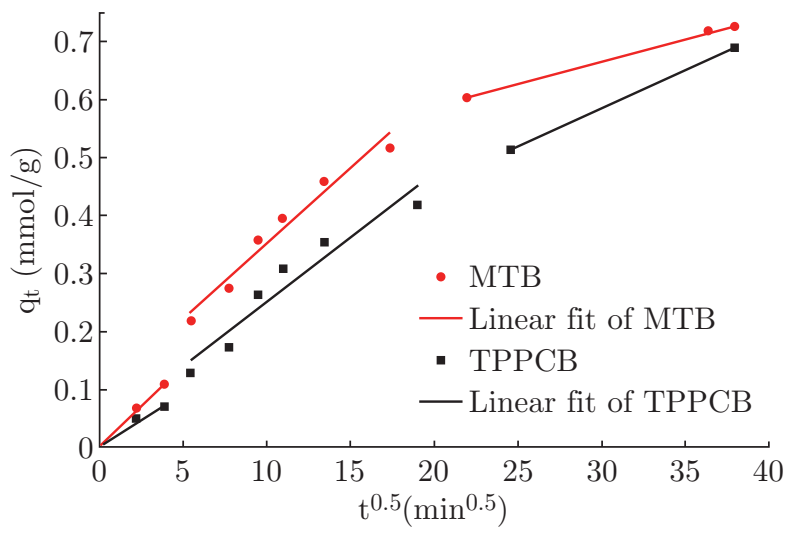

(d)

Fig. 7: (a) Removal of RB19 by using MTB and TPPCB; (b) Pseudo first order modelling; (c) Pseudo second order modelling and (d) Intraparticle diffusion modelling. Adsorption conditions: temperature of $37^{\circ} \mathrm{C}, 50 \mathrm{ml}$ of $1.5 \mathrm{mM}$ adsorbate at $\mathrm{pH} 3,0.1 \mathrm{~g}$ adsorbent, shaking speed of $140 \mathrm{rpm}$

Table 3: Kinetics parameters of the adsorption of RB19 by MTB and TPPCB

\begin{tabular}{|c|c|c|c|c|c|c|}
\hline & \multicolumn{3}{|c|}{ Pseudo first order } & \multicolumn{3}{|c|}{ Pseudo second order } \\
\hline & $k_{1}\left(\min ^{-1}\right)$ & $q_{e}\left(\mathrm{mmol} \mathrm{g}^{-1}\right)$ & $\mathrm{R}^{2 \mathrm{a}}$ & $k_{2}\left(\mathrm{~g}^{-1} \mathrm{mmol}^{-1} \min ^{-1}\right)$ & $q_{e}\left(\mathrm{mmol} \mathrm{g}^{-1}\right)$ & $\mathrm{R}^{2 \mathrm{a}}$ \\
\hline MTB & 0.0030 & 0.5811 & 0.9791 & 0.0157 & 0.7477 & 0.9894 \\
\hline TPPCB & 0.0026 & 0.6157 & 0.9794 & 0.0102 & 0.7028 & 0.9647 \\
\hline \multicolumn{7}{|c|}{ Intraparticle diffusion } \\
\hline & \multicolumn{3}{|c|}{ MTB } & \multicolumn{3}{|c|}{ ТРРСВ } \\
\hline $\begin{array}{c}k_{i} \\
\left(\mathrm{mmol} \mathrm{g}{ }^{-1} \min ^{-0.5}\right)\end{array}$ & 0.0283 & 0.0261 & 0.0078 & 0.0182 & 0.0221 & 0.013 \\
\hline $\mathrm{R}^{2 a}$ & 0.9955 & 0.9549 & 0.9975 & 0.9643 & 0.9016 & - \\
\hline
\end{tabular}

a _ correlation coefficient

\section{Conclusions and Future Works}

An easy and simple method to prepare physically and chemically stable CHI beads has been achieved by a freeze-drying process. The encapsulation of live bacterium, L. casei, is also suc- 
cessfully achieved with a similar process. Freeze dried blank CHI or TPPCB shows the complete decolorisation of RB19 at a concentration of $0.15 \mathrm{mM}$ within 2 hours at a $\mathrm{pH}$ of 3 and temperature of $37^{\circ} \mathrm{C}$. L. casei encapsulated CHI beads or MTB shows faster kinetics than TPPCB upon adsorbing $1.5 \mathrm{mM}$ of RB19 at $\mathrm{pH} 3$ and temperature of $37^{\circ} \mathrm{C}$. By fitting several kinetic models to the adsorption process, both MTB and TPPCB partially follow the intraparticle diffusion model. MTB follows the pseudo second order in the mid-stage of adsorption while TPPCB follows the pseudo first order. A more comprehensive kinetics study and isotherm modelling will need to be performed to provide a better understanding of the adsorption mechanism and estimate the maximum adsorption capacity of the adsorbents.

\section{References}

[1] R. Patel, S. Suresh, Kinetic and equilibrium studies on the biosorption of reactive black 5 dye by aspergillus foetidus, Bioresour. Technol., 99, 2008, 51-58

[2] A. Mahwish, Biosorption of reactive dyes: A review, Water Air Soil Pollut., 223, 2012, 2417-2435

[3] X. Jiang, Y. M. Sun, L. Liu, S. G. Wang, X. Z. Tian, Adsorption of C.I. Reactive Blue 19 from aqueous solutions by porous particles of the grafted chitosan, Chem. Eng. J., 235, 2014, 151-157

[4] R. Khan, P. Bhawana, M. H. Fulekar, Microbial decolorization and degradation of synthetic dyes: A review, Rev. Environ. Sci. Biotechnol., 12, 2013, 75-97

[5] A. Saeed, M. Iqbal, S. I. Zafar, Immobilization of trichoderma viride for enhanced methylene blue biosorption: Batch and column studies, J. Hazard. Mater, 168, 2009, 406-415

[6] D. C. Vodnar, C. Socaciu, A. M. Rotar, Stanila A. Morphology, FTIR fingerprint and survivability of encapsulated lactic bacteria (streptococcus thermophilus and lactobacillus delbrueckii subsp. bulgaricus) in simulated gastric juice and intestinal juice, Int. J. Food Sci. Technol., 45, 2010, $2345-2351$

[7] P. Schar-Zammaretti, J. Ubbink, The cell wall of lactic acid bacteria: Surface constituents and macromolecular conformations, Biophys. J., 85, 2003, 4076-4092

[8] A. Bhatnagar, M. Sillanpää, Applications of chitin- and chitosan-derivatives for the detoxification of water and wastewater - A short review, Adv. Colloid Interface, 152, 2009, 26-38

[9] S. Lagergren, About the theory of so-called adsorption of soluble substances, Kungliga Svenska Vetenskapsakademiens Handlingar, 24, 1898, 1-39

[10] Y. S. Ho, G. McKay, A comparison of chemisorption kinetic models applied to pollutant removal on various sorbents, Process Saf. Environ., 76, 1998, 332-340

[11] W. J. Weber, J. C. Morris, Kinetics of adsorption on carbon from solution, J. Sanit. Eng. Div. Proc. A.S.C.E., 89, 1963a, 31-59 